\section{Assisted sedation for percutaneous endoscopic gastrostomy procedure in sick patients in a developing country}

\section{Somchai Amornyotin, Wiyada Chalayonnavin, Siriporn Kongphlay}

Siriraj GI Endoscopy Center and Department of Anesthesiology, Faculty of Medicine, Siriraj Hospital, Mahidol University, Bangkok, Thailand

\section{Abstract}

In a developing country, the percutaneous endoscopic gastrostomy (PEG) procedure is being performed at an increasing rate. However, there is sparse information on the sedation for this procedure. The aim of our study was to evaluate the clinical efficacy of assisted sedation compared with general anesthesia for the PEG procedure in patients in a teaching hospital in Thailand. We undertook a retrospective review of records of patients who underwent PEG procedures from October 2006 to September 2008. All patients with ASA physical status III and IV were classified into two groups according to the type of anesthetic technique: Group $\mathrm{S}$, intravenous sedation (IVS); and Group G, general anesthesia (GA). The primary outcome of the study was the successful completion of the procedure. The secondary outcomes were sedation/anesthesiarelated complications during and immediately after the procedure.

One hundred and eighty-four patients were enrolled with 116 (M, 52; mean age, 71.4 \pm 15.8 years) in Group $\mathrm{S}$ and 68 (M, 37; mean age, $67.8 \pm 18.5$ years) in Group G. There were no significant differences between the two groups in age, gender, weight, and duration of procedure. All patients in both groups concluded with the successful completion of the procedure. Overall adverse events in group $S$ occurred significantly less commonly than in group $\mathrm{G}(\mathrm{P}=0.003)$. All complications were treated easily with no adverse sequelae. We concluded that in the setting of the developing country, assisted sedation for the PEG procedure in patients by trained anesthetic personnel with appropriate monitoring was safe and effective.

\section{Introduction}

Percutaneous endoscospic gastrostomy (PEG) has become the procedure of choice for enteral feedings in patients with a functioning gastrointestinal tract who need long-term enteral feeding when oral access is impossible. ${ }^{1,2}$ The procedure is safe and simple technically, and the tube feeding can be handled easily in a nursing home or even by caregivers at home. ${ }^{3,4}$ PEG has replaced the surgical gastrostomy procedure because of its lower cost and shorter recovery time. Many patients requiring PEG are older, frail, malnourished, and have a significant comorbidity. PEG insertion is an invasive procedure requiring both endoscopy and sedation. It usually carries a risk of high mortality rates in the early postinsertion period, with 30-day mortality rates varying between $4 \%$ and $26 \%{ }^{5}$ Furthermore, there is a substantial risk of morbidity, especially from aspiration pneumonia (1.7\%-2.7\%), ${ }^{6,7}$ and PEGsite infection (3\%-11\%), ${ }^{8,9}$

Anesthesia consultation before the procedure may be needed. Fluid and electrolyte disorders should be corrected and any infection treated. Antibiotic prophylaxis is recommended because of infection risks. Ideally, PEG should be performed in an operating room (OR). In practice, however, most procedures are performed in an endoscopy room, with special precautions taken. The type of anesthesia used is decided according to the patient's medical condition and the anesthesiologist's preference. Local anesthesia with mild sedation can be used, but to assure better patient comfort during this procedure, short-term general anesthesia is preferred. In our hospital, the majority of PEG procedures were undertaken with assisted sedation. ${ }^{10}$

We conducted a retrospective study to compare the clinical efficacy of assisted sedation and general anesthesia for the PEG procedure in a tertiary-care teaching hospital in a developing country to determine the safety and efficacy of assisted sedation administrated by trained anesthetic personnel for the PEG procedure.

\section{Materials and Methods}

\section{Patients}

The patients who underwent the PEG procedure at Siriraj GI Endoscopy Center, Faculty of Medicine, Siriraj Hospital between October 2006 and September 2008 were enrolled in our study. Patients with ASA physical status III and IV were included. The PEG procedures performed in the intensive care units and in operating rooms as well as the procedures performed without sedation and general anesthesia were excluded.

\section{Study design}

This study was a retrospective descriptive study. All sick patients were classified into two
Correspondence: Somchai Amornyotin, Department of Anesthesiology, Faculty of Medicine, Siriraj Hospital, Mahidol University, Bangkok, Thailand. E-mail: sisam@mahidol.ac.th

Key words: percutaneous endoscopic gastrostomy, intravenous sedation, general anesthesia.

Conflict of interest: the authors report no conflicts of interest.

Received for publication: 13 November 2009.

Revision received: 20 January 2010.

Accepted for publication: 24 January 2010.

This work is licensed under a Creative Commons Attribution 3.0 License (by-nc 3.0).

(C) Copyright S. Amornyotin et al., 2010

Licensee PAGEPress, Italy

Gastroenterology Insights 2010; 2:e5

doi:10.4081/gi.2010.e5

groups according to the type of anesthetic technique. In group S, PEG was done by using the intravenous sedation (IVS) technique. In group G, PEG was performed with the general anesthesia (GA) technique. The primary outcome of the study was the successful completion of the procedure. The secondary outcomes were sedation/anesthesia-related complications during and immediately after the procedure.

\section{Endoscopy procedure}

All PEG procedures were done using an Olympus video esophagogastroduodenoscope (GIF-Q 180, Olympus Corporation, Tokyo, Japan). After completion of the procedure, admission into the inpatient hospital service was arranged to rule out post-PEG complications, which were defined using criteria as described by DeLegge. ${ }^{11}$

\section{Anesthesia-related procedure}

The patients were monitored with noninvasive blood pressure, ECG, and pulse oximetry. IVS and GA techniques were the main anesthesia methods employed. The choice of these techniques is dependent upon the patient's medical condition and the familiarity of the anesthesiologist with the particular case. The type, dose, and combination of sedative and analgesic agents were assessed. Complications such as hypotension, bradycardia, hypoxia $\left(\mathrm{SpO}_{2}<90 \%\right)$, or airway obstruction were recorded.

\section{Statistical analysis}

Results were expressed as mean \pm SD or percentage (\%), when appropriate. Comparisons between sedation and general anesthesia groups were made by using with $\chi^{2}$-tests (i.e. for categorical variables), $\chi^{2}$-tests for trend (for 
ordinal variables), and two-sample independent $t$-test (for continuous variables). The statistical software package SPSS for Window Version 11 (SPSS Inc., Chicago, IL, USA) was used to analyze the data. All statistical comparisons were made at the two-sided 5\% level of significance.

\section{Results}

Two hundred and thirty-nine PEG procedures (114 men, 125 women; mean age 69.8 years) were performed during the study period. Of these, 184 patients had ASA physical status III and IV and were enrolled in the study. One hundred and sixteen patients (52 men, 64 women; mean age $71.4 \pm 15.8$ years) were classified in group $\mathrm{S}$ (sedation) and 68 patients (37 men, 31 women; mean age $67.8 \pm 18.5$ years) in group $\mathrm{G}$ (general anesthesia).

The characteristics of the group $\mathrm{S}$ and $\mathrm{G}$ populations were compared. There were no statistically significant differences between the two groups in age, gender, weight, ASA physical status, or procedure time. Characteristics of patients and duration of PEG procedure were summarized in Table 1 Indications for PEG are also shown in Table 1. More patients underwent PEG for dementia, and oral, laryngeal, and esophageal malignancy in group $\mathrm{S}$ than in group $\mathrm{G}$. In contrast, more patients underwent PEG for brain anoxia in group $\mathrm{G}$ than in group $\mathrm{S}$.

In group $S, 88$ patients (75.9\%) underwent the procedure using the combination of IVS and the topical pharyngeal anesthesia technique, and 28 patients were sedated by using the IVS technique. In group G, all patients were anesthetized by using the GA technique and either a tracheostomy tube (91.2\%) or tracheal tube $(8.8 \%)$. All anesthesia was given by the staff anesthesiologist, or the anesthetic personnel directly supervised by the staff anesthesiologist who was physically present in the endoscopy room. Anesthetic personnel included residents in the Anesthesiology Residency Program and anesthetic nurses who are well trained in general anesthesia, intravenous sedation, airway management including intubation, and cardiopulmonary resuscitation.

Cardiovascular monitoring, including blood pressure measurements, electrocardiogram, heart and respiratory rate, and oxygen saturation, was performed. No pre-medications were used before the procedure. End-tidal carbon dioxide $\left(\mathrm{CO}_{2}\right)$ monitoring was not used during sedation/anesthesia. In group $\mathrm{S}$, the patients were oxygenated with $100 \% 0_{2}$ via a nasal cannula and sedated by well-trained anesthetic personnel, directly supervised by a staff anesthesiologist in the endoscopy room. All of these patients were sedated to a moderate (conscious) sedation level, according to guidelines

Table 1. Characteristics of patients, duration and indications of PEG procedur.

\begin{tabular}{lccc} 
& $\begin{array}{c}\text { Sedation } \\
(\mathrm{n}=116)\end{array}$ & $\begin{array}{c}\text { General anesthesia } \\
(\mathrm{n}=68)\end{array}$ & P \\
Age (yr) (mean, SD) & $71.4(15.8)$ & $67.8(18.5)$ & 0.34 \\
Gender (n, \%) & & & \\
$\quad$ Male & $52(44.8)$ & $37(54.4)$ & 0.21 \\
Female & $64(55.2)$ & $31(45.6)$ & \\
Weight (kg) (mean, SD) & $48.7(9.4)$ & $50.2(7.9)$ & 0.39 \\
ASA physical status (n, \%) & & & 0.12 \\
III & $114(98.3)$ & $64(94.1)$ & \\
IV & $2(1.7)$ & $4(5.9)$ & \\
Duration of procedure (min) (mean, SD) & $26.6(7.5)$ & $28.5(5.9)$ & 0.07 \\
Indications (n, \%) & & & $0.009 *$ \\
Cerebrovascular accident & $34(29.3)$ & $21(30.9)$ & \\
Dementia & $30(25.9)$ & $8(11.8)$ & \\
Oral, laryngeal, and esophageal malignancy & $19(16.4)$ & $7(10.3)$ & \\
Prolonged nasogastric tube insertion & $10(8.6)$ & $8(11.8)$ & \\
Brain anoxia & $2(1.7)$ & $10(14.7)$ & \\
Parkinsonism & $7(6.0)$ & $4(5.9)$ & \\
Miscellaneous & $14(12.1)$ & $10(14.7)$ & \\
\hline
\end{tabular}

* Considered statistically significant.

Table 2. Success rate and anesthetic agents used in both groups.

\begin{tabular}{lccc} 
& $\begin{array}{r}\text { Sedation } \\
(\mathrm{n}=116)\end{array}$ & $\begin{array}{r}\text { General anesthesia } \\
(\mathrm{n}=68)\end{array}$ & P \\
Success rate (n, \%) & $116(100.0)$ & $68(100.0)$ & 1.00 \\
Sedative agents (n, \%; mean, SD) & & & \\
$\quad$ Fentanyl (mg/kg) & $116(100.0)$ & $66(97.1)$ & 0.06 \\
& $0.001(0.00)$ & $0.001(0.00)$ & \\
Propofol (mg/kg) & $102(87.9)$ & $40(58.8)$ & $<0.001^{*}$ \\
& $0.85(0.58)$ & $1.12(0.39)$ & \\
Midazolam (mg/kg) & $94(81.0)$ & $21(30.9)$ & $<0.001^{*}$ \\
Ketamine (mg/kg) & $0.02(0.01)$ & $0.03(0.01)$ & 0.30 \\
& $5(4.3)$ & $1(1.5)$ & \\
Inhalation agents (n, \%) & $0.63(0.32)$ & 0.5 & \\
Sevoflurane & & & \\
Isoflurane & 0 & $33(48.5)$ & \\
Desflurane & 0 & $4(5.9)$ & \\
Muscle relaxation agents (n, \%) & 0 & $2(2.9)$ & \\
Atracurium & & & \\
Vecuronium & 0 & $7(10.3)$ & \\
\hline
\end{tabular}

* Considered statistically significant.

Table 3. Sedation/anesthesia-related adverse events (n, \%).

\begin{tabular}{lccc} 
Adverse events & $\begin{array}{c}\text { Sedation } \\
(\mathbf{n}=116)\end{array}$ & $\begin{array}{c}\text { General anesthesia } \\
(\mathrm{n}=68)\end{array}$ & $0.003^{*}$ \\
Overall & $19(16.4)$ & $24(35.3)$ & 0.28 \\
$\begin{array}{l}\text { Respiratory } \\
\text { Hypoxia }\end{array}$ & $2(1.7)$ & 0 & 0.28 \\
$\quad\left(\mathrm{SpO}_{2}<90 \%\right)$ & $2(1.7)$ & 0 & $0.001^{*}$ \\
Cardiovascular & $17(14.7)$ & $24(35.3)$ & $0.001^{*}$ \\
Hypotension & $16(13.8)$ & $24(35.3)$ & 0.44 \\
Bradycardia & $1(0.9)$ & 0 & \\
\hline
\end{tabular}

*Considered statistically significant.

of the American Society of Anesthesiologists. ${ }^{12}$ In group $\mathrm{G}$, the patients were oxygenated with $50 \% \mathrm{O}_{2}$ with controlled ventilation. In addition, they were anesthetized with sedative drugs and inhalation agents with or without muscle relaxants.
Table 2 shows the success rate and anesthetic agents used in the sedation and GA groups. All patients in both groups concluded with the successful completion of the procedure. Of the sedative agents, fentanyl, propofol, and midazolam were used frequently in the 
two groups. Most of them were used in combination with either two or three other agents. There were no statistically significant differences between the two groups in the mean dose of fentanyl and ketamine. However, the mean dose of propofol and midazolam in group $\mathrm{S}$ was significantly lower than in group $\mathrm{G}$ $(\mathrm{P}<0.001)$. In group $\mathrm{S}$, the combination of fentanyl, propofol, and midazolam (69.9\%) was the most common combination of intravenous sedative agents used. In group G, sevoflurane was the most common inhalation agent, and atracurium was the most common muscle relaxant drug used.

Overall, 19 patients (16.4\%) in group $\mathrm{S}$ and 24 patients (35.3\%) in group $\mathrm{G}$ experienced adverse events. In group $\mathrm{S}$, a respiratory adverse event (hypoxia: $\mathrm{SpO}_{2}<90 \%$ ) occurred in $1.7 \%$ of patients, and comprised $10.5 \%$ of all adverse events, all of which were under the care of an anesthesiologist. In group $\mathrm{G}$, there were no respiratory adverse events. Additionally, cardiovascular adverse events arose in $14.7 \%$ and $35.3 \%$ of patients in groups $\mathrm{S}$ and $\mathrm{G}$, respectively. They mainly consisted of hypotension $(94.1 \%$ in group $\mathrm{S}$ and $100 \%$ in group G). Interestingly, the cardiovascular adverse events in group $\mathrm{S}$ occurred significantly less often than in group $\mathrm{G}(\mathrm{P}=0.001$, Table $3)$. No procedures were aborted as a result of insufficient sedation or complications of sedation/anesthesia. There were no procedurerelated complications in either group.

\section{Discussion}

PEG was introduced in 1980 by Ponsky and his colleagues. ${ }^{13-15}$ Since then, it has gained wide acceptance as a safe and efficient method of providing enteral alimentation in patients who cannot swallow..$^{15}$ The advantages of PEG over the traditional open gastrostomy approach are unequivocal and relate primarily to the avoidance of a laparotomy. Use of PEG results in reduced procedure time, cost, recovery time, and complications. ${ }^{14}$ Many physicians now consider PEG to be the method of choice for feeding patients with dysphagia because of either neurological disorders or head and neck malignancies. This procedure is a minimally invasive one, with low procedure-related major complications and mortality rates.

PEG is an essential procedure among gastrointestinal (GI) abnormality treatments, even in our institution where we observe an increase in the number of these procedures every year. Therefore, it is mandatory to standardize a safe, easy, well-tolerated anesthesiological procedure, which is feasible in the GI endoscopy unit. In our previous experience, we have noted that topical anesthesia alone is not sufficient for pain-free procedures. In contrast, general anesthesia, which may be of benefit for patient's and endoscopist's comfort, may be difficult to administer especially in comorbidity patients. In addition, the lack of experience in anesthesia care among endoscopy personnel might increase the risk of complications.

Propofol, combined with short-acting benzodiazepine with or without fentanyl, has been used in several GI endoscopic procedures. In this study, were have shown that assisted sedation with low-dose midazolam and fentanyl, and/or low-dose propofol, is safe and well tolerated by the patient. Furthermore, it is well accepted by endoscopists. No patients enrolled in our study needed to be resuscitated during the PEG procedure. All patients could be discharged to the ward within 30 minutes after the procedure, and this discharge time was not correlated with age, ASA physical status, and total sedative doses.

Patients were breathing spontaneously; however, oxygen saturation was always over $95 \%$, and age, ASA physical status, and the combination of sedative agents did not negatively influence this parameter. In addition, heart rate was only partially affected. Sedation is performed to ensure the patient's safety, to minimize physical discomfort or pain, to provide analgesia and procedural amnesia, to control behavior during the procedure, and to return the patient to pretreatment level of consciousness. The amount of sedation required depends on the patient's physical status and age. Propofol is widely employed for anesthesia outside the OR because it is easy to use, has a good safety and efficacy profile owing to its quick onset of action, rapid metabolism, and significantly shorter recovery time, and it has some antiemetic effects. ${ }^{16-18}$ All of these properties are useful in endoscopic procedures.

Propofol is known to decrease blood pressure in young and old people. ${ }^{17}$ This effect was noticed in our study. In particular for diastolic blood pressure, the difference between younger and older patients over time was significant. However, severe hypotension that required resuscitation treatment did not develop for age or ASA physical status groups. Total dose was inversely correlated with the patient's age. The influence of age on the propofol dose in decreasing systolic blood pressure has been described already. ${ }^{19}$

Low-dose midazolam as well as ketamine, combined with low-dose fentanyl and propofol, did not prolong recovery time. Moreover, ketamine accompanied by these agents resulted in stable hemodynamics and did not produce emergence reactions or hallucinations. Consequently, sick patients (ASA III-IV or comorbidities) may be sedated utilizing this combination technique. However, the combined group studied is small; therefore, more patients are needed.
We used only standard monitoring in our study, including an assessment of blood pressure, pulse rate, respiratory rate, and pulse oximetry, as well as the electrocardiogram. We detected a relatively high overall rate of adverse events in both groups. This rate is higher than that commonly reported, and there may be several explanations for this. We used these criteria in defining adverse events: hypo/hypertension and brady-/tachycardia measured as the changes of blood pressure and heart rate of more than $20 \%$ of base line values. Hypoxia was defined as oxygen saturation $<90 \%$. Hypercapnia (ETCO $2>50 \mathrm{mmHg}$ ) could not be detected directly in our study. If only significant respiratory adverse events such as prolonged desaturation or apnea are counted, the adverse event rate is $1.2 \%$, which corresponds to the previously published studies. ${ }^{20}$ Moreover, if only serious adverse events are included, the adverse event rate is $0.6 \%$ in group $\mathrm{S}$ and zero in group $\mathrm{G}$. Interestingly, we found that all adverse events occurred in the propofol-related group.

In one prospective clinical trial, 100 patients undergoing EGD or colonoscopy were sedated with low-dose propofol, midazolam, and fentanyl (or pethidine). This combination produced a moderate level of sedation. There was no serious adverse event. ${ }^{21}$ We believe that the appropriate selection of patients for sedation is very important for everyday practice and will most likely reduce the rate of adverse events. Finally, the use of pulse oximetry to monitor hypoxemia is important, especially in cases when supplemental oxygen is administered.

Data from our previous study ${ }^{22}$ showed that both patient and endoscopist satisfaction was higher in sedated patients than in nonsedated patients. The use of sedation was the major determinant of patient satisfaction and willingness to repeat the procedure. However, assisted sedation contributed to an increased recovery room time. Among all of these benefits, it is advantageous to identify the particular factors that might encourage patients to undergo the PEG procedure with assisted sedation. Moreover, the present study showed that PEG procedures in sick patients can be performed safely and effectively with a lower complication rate under intravenous sedation. We think that low-dose fentanyl combined with low-dose midazolam is the ideal combination. In our hospital, intravenous sedation was extensively used for PEG procedures. ${ }^{10}$ However, this is not widespread in the district community hospital.

Limitations of our study exist. First, there is the wide range in age of the patients we studied. Drug requirements, recovery time, and side effects can be related to patient's age. Second, inaccurate and incomplete documentation of certain measures, as occurs with many chart reviews, also occurred in this 
study. Third, the limitation of monitoring, such as of end-tidal carbon dioxide, could result in a lower rate of adverse events. Fourth, the PEG procedure was performed by a variety of endoscopists including fellows in training. Therefore, the varied experience may have biased the result, including the successful completion rate and complication rate. However, the effect of this may be small given the high successful completion of the procedures and equal amount of time used for the completion of the procedures in both groups. Fifth, more patients with dementia, and oral, laryngeal, and esophageal malignancies in group $\mathrm{S}$, and more patients with brain anoxia in group $\mathrm{G}$ underwent the PEG procedures. The indications for PEG procedures were not indifferent. Finally, anesthesiologists define complications differently. Overall, despite these limitations we are confident that these findings are generalizable to the practice of using the PEG procedure with any type of assisted sedation. Furthermore, because the serious complications in our series were low, further studies in larger prospective groups of patients are needed.

In conclusion, we report here the performance and clinical efficacy of the assisted sedation regimen, utilizing the anesthesiologist or anesthetic personnel with appropriate basic monitoring for the PEG procedure, in patients with ASA III and IV in a unit outside OR, from a tertiary-care teaching hospital in a developing country. The findings of the present study also showed that the PEG procedure done with assisted sedation was as safe and effective as that done with GA. The combination of lowdose fentanyl and midazolam may be most beneficial.

\section{References}

1. Tham TCK, Taitelbaum G, Carr-Locke DL. Percutaneous endoscopic gastrostomies: are they being done for the right reasons? Qual J Med 1997;90:495-6.
2. Kirby DF, Delegge MH, Fleming CR. American Gastroenterological Association technical review on tube feeding for enteral nutrition. Gastroenterology 1995;108: 1282-301.

3. Loeser C, von Herz U, Kuchler T, et al. Quality of life and nutritional state in patients on home enteral tube feeding. Nutrition 2003;19:605-11.

4. Hebuterne X, Bozzetti F, Moreno Villares $\mathrm{JM}$, et al. Home enteral nutrition in adults: a European multicentre survey. Clin Nutr 2003;22:261-6.

5. Abuksis G, Mor M, Segal N, et al. Percutaneous endoscopic gastrostomy: high mortality rates in hospitalized patients. Am J Gastroenterol 2000;95:12832 .

6. Gencosmanoglu R, Koc D, Tozun N. Percutaneous endoscopic gastrostomy: results of 115 cases. Hepatogastroentero$\log 2003 ; 50: 886-8$.

7. Carnes ML, Sabol DA, DeLegge M. Does the presence of esophagitis prior to PEG placement increase the risk for aspiration pneumonia? Dig Dis Sci 2004;49:1798-802.

8. Ahmad I, Mouncher A, Abdoolah A, et al. Antibiotic prophylaxis for percutaneous endoscopic gastrostomy: a prospective, randomized, double blind trial. Aliment Pharmacol Ther 2003;18:209-15.

9. Saadeddin A, Freshwater DA, Fisher NC, et al. Antibiotic prophylaxis for percutaneous endoscopic gastrostomy for nonmalignant conditions: a double blind prospective randomized controlled trial. Aliment Pharmacol Ther 2005;22:565-70.

10. Amornyotin S, Prakanrattana U, Chalayonnavin W, et al. Anesthesia for percutaneous endoscopic gastrostomy in Siriraj Hospital. Thai J Anesth 2009;35:39-47.

11. DeLegge MH. Endoscopic enteral access for enteral nutrition. Am Soc Gastrointest Endosc 2007;doi:10.1016/j.clinup.2007.08. 001.

12. American Society of Anesthesiologists. Practice guidelines for sedation and analgesia by nonanesthesiologists. An update report by the ASA Task Force on Sedation and Analgesia by Nonanesthesiologists. Anesthesiology 2002;96:1004-17.

13. Gauderer MWL, Ponsky JL, Izant RJ Jr. Gastrostomy without laparotomy: a percutaneous endoscopic technique. J Pediatr Surg 1980;15:872-5.

14. Ponsky JL, Gauderer MWL. Percutaneous endoscopic gastrostomy: a nonoperative technique for feeding gastrostomy. Gastrointest Endosc 1981;27:9-11.

15. Ponsky JL, Gauderer MWL, Stellato TA. Percutaneous endoscopic gastrostomy: review of 150 cases. Arch Surg 1983; 118:913-4.

16. Gasparovic S, Rustemovic N, Opacic M, et al. Clinical analysis of propofol deep sedation for 1,104 patients undergoing gastrointestinal endoscopic procedures: A three year prospective study. World J Gastroenterol 2006;12:327-30.

17. Heuss LT, Inauen W. The dawning of a new sedative: Propofol in gastrointestinal endoscopy. Digestion 2004;69:20-6.

18. Chutkan R, Cohen J, Abedi M, et al. Training guideline for use of propofol in gastrointestinal endoscopy. Gastrointest Endosc 2004;60:167-72.

19. Kazama T, Ikeda K, Morita K, et al. Comparison of the effect-site $\mathrm{k}(\mathrm{e} 0) \mathrm{s}$ of propofol for blood pressure and EEG bispectral index in elderly and younger patients. Anesthesiology 1999;90:1517-27.

20. Basells F, Wyllie R, Kay M, et al. Use of conscious sedation for lower and upper gastrointestinal endoscopic examination in children, adolescents, and young adults: a twelve-year review. Gastrointest Endosc 1997;45:375-80.

21. Cohen LB, Hightower CD, Wood DA, et al. Moderate level sedation during endoscopy: a prospective study using low-dose propofol, meperidine/fentanyl, and midazolam. Gastrointest Endosc 2004;58:795-803.

22. Amornyotin S, Lertakayamanee N, Wongyingsinn $\mathrm{M}$, et al. The effectiveness of intravenous sedation in diagnostic upper gastrointestinal endoscopy. J Med Assoc Thai 2007;90:301-6. 